\title{
Outbreak of hepatitis B in Sabarkantha district of Gujarat: A case-control study
}

Background: An outbreak of jaundice syndrome was reported from Sabarkantha district of Gujarat in February 2009. Initial investigation of serum specimens collected from the patients was positive for hepatitis B. The outbreak was investigated thoroughly. Materials and Methods: A confirmed case definition was made as a person residing in Sabarkantha district who developed jaundice symptoms with SGPT $>400 \mathrm{IU}$ and tested positive for HBsAg. A case-control study was conducted to identify the source of infection and to recommend public health measures. Results: Five-hundred and twenty-eight cases were reported of which, 93 died (Case fatality rate $=17.6 \%$ ). Majority $(61 \%$ ) were males, and more than $77 \%$ were in the age group of $12-45$ years. Eighty-four percent reported history of taking injections/needle prick in the last 6 months. In the case-control study there was strong association between cases and history of injection/needle prick (OR $=42.17$ ). Hepatitis $B$ mass vaccination was carried out to control the outbreak. Conclusion: This outbreak of hepatitis-B was associated with unsafe injection practices in private clinics of Sabarkantha district. Sensitization workshop for safe injection practices and waste management held among doctors. Legal actions were taken against the private practitioners involved in these unsafe injection practices.

Key words: Hepatitis B outbreak, jaundice, mass vaccination, unsafe injection practice

Bisworanjan Dash, Rashmi Ranjan Das ${ }^{1}$

Epidemiologist, National Centre for Disease Control, New Delhi,

${ }^{1}$ Department of Pediatrics, All India Institute of Medical Science, New Delhi, India

Address for the Correspondence:

Dr. Bisworanjan Dash, Epidemiologist, National Centre for Disease Control, 22-Shamnath Marg, New Delhi - 54, India. E-mail: drbiswo@gmail.com

\begin{tabular}{|l|}
\hline Access this article online \\
\hline Website: www.ijmedph.org \\
\hline DOI: 10.4103/2230-8598.115188 \\
\hline Quick response code: \\
\hline
\end{tabular}

\section{INTRODUCTION}

Hepatitis B infection can cause both acute and chronic liver diseases. HBV has a worldwide spread, with an estimated 400 million persons chronically infected. The areas of highest prevalence of HBV infection are sub-Saharan Africa, China, and parts of the Middle East..$^{[1,2]}$ An estimated number of 600,000 persons die each year due to the acute or chronic consequences of hepatitis B. India comes under the 'intermediate' endemicity $(2-7 \%)$ zone as the mean national surface antigen carrier rate is around $4.7 \% \cdot \cdot^{[1,2]}$ Assuming the population of India to be around 900 million, then there will be an estimation of 42.5 million carriers in the country. HBV infection in selected high risk group in India ranges between 12 and $74 \%$.

HBV is present in high concentrations in blood, serum, and serous exudates and in moderate concentrations in saliva, vaginal fluid, and semen; efficient transmission occurs through blood exposure and sexual contact. Risk factors for HBV infection include intravenous acquisition by drugs or blood products, acupuncture or tattoos, sexual contact, institutional care, and intimate contact with carriers. ${ }^{[1,2]}$ HBV has eight genotypes (A-H). A is pandemic, B, and $\mathrm{C}$ are prevalent in Asia. ${ }^{[2]}$ In addition, genetic variants have become resistant to antiviral agents. After infection, the incubation period ranges from 45 to 160 days, with a mean of about 120 days.

An increased incidence of jaundice syndrome was reported from Modasa taluka and few cases from surrounding areas of Sabarkantha district of Gujarat, India on $10^{\text {th }}$ February 2009. Immediately a team from local medical college and a central team including ours visited Madosa block and reviewed the situation. Serum samples were collected from the affected subjects and were found to be positive for HBsAg, which established the outbreak to be due to of hepatitis B. The objectives were to investigate the cause of hepatitis outbreak, and to recommend control and preventive measures in that area. 


\section{Evaluation of the outbreak}

After discussion with the state and district health authorities including hospital authorities, the team visited the hospital wards and did the clinical assessment of admitted cases. Study of the hospital records were also carried out. After that, visit of the affected areas of Sabarkantha district was made. At the outbreak site, interview of the patients or relatives of deceased were carried out. Finally, collection of the clinical specimens for laboratory investigations followed by discussions with the local leaders and villagers were conducted.

\section{Magnitude of the problem}

The team was informed that the outbreak was first noticed on February 10, 2009. A total of 528 cases were reported to the district authorities between $26^{\text {th }}$ January and $19^{\text {th }}$ June 2009 . Most of the cases were reported from Modasa block $(n=195)$, followed by Idar $(n=155)$, Himmatnagar $(n=55)$, Meghraj $(n=38)$, Bayad $(n=25)$, and Bhiloda $(n=19)$. Of these, 93 had died (case fatality $=17.6 \%$ ). Number of cases and deaths peaked in week ending $23^{\text {rd }}$ February 2009 (fourth week of the outbreak), and declined there-after [Figure 1]. Most cases occurred in age groups 13-45 years, and 324 (61\%) cases were male [Table 1].

\section{Clinical presentation of the cases}

The cases initially presented with a short history of fever, nausea/ vomiting, abdominal pain/discomfort. They soon developed jaundice, followed by irritability, altered behavior leading to coma. Some patients have developed renal failure. Most patients had history of having received injections in the past few months, though no documentation of this is available. The outbreak was traced to a particular clinic that was practicing unsafe injection administration.

\section{Laboratory investigations}

The patients have been found to have high serum bilirubin and very high SGPT levels. Most patients have tested positive for HBsAg. A few also tested for IgM anti-HBc and showed a positive result. Hepatitis $\mathrm{C}$ was also simultaneously tested has been tested in a few samples, but found negative.

\section{Case management}

Symptomatic and supportive treatment was given to the patients in the form of IV fluids, antibiotics, antipyretics, and mannitol, etc., The local hospital as well as the medical college set up isolation wards, where most of the cases were managed. Standard protocol was prepared and followed for treatment of the patients.

\section{Analysis of the outbreak}

Our NCDC team conducted a case-control study taking injection history of 13 cases and 26 controls. A systematic sampling method was used in a total sample of 260 line listing of cases. Every twentieth number of these cases was interviewed regarding the exposure history to need prick (accidental or during blood sampling for routine investigation) or taking injection for underlying health condition in last 6 months. Controls were household contacts of these cases, and to increase the power of the test, two times the number of the cases were selected as controls and interviewed in their respective houses regarding similar history mentioned above for the cases. Eighty-four percent (84\%) of the cases versus $13 \%$ of the controls selected had history of exposure (injection) within the incubation period of 6 months (OR $(95 \% \mathrm{CI}) ; 42.17$ (6.13, 289.95); $P=0.000007$ ) [Table 2]. This proves that hepatitis B among the affected cases was caused by unsafe injection practices.

\section{Recommendations made}

1. Taking appropriate action for the prevention of unsafe (injection) practices among the medical fraternity.

2. Continuing the health awareness campaign about the unsafe injection practices among the community as well as medical and paramedical workers.

3. Mass vaccination with three doses of hepatitis $B$ vaccine.

3. Observing the side effects and benefits of antiviral therapy started on hepatitis B patients.

4. Counseling of sexual partners of the affected cases and advise to practice barrier method of contraception for a period of 6 weeks at least after the spouse is discharged from the hospital.

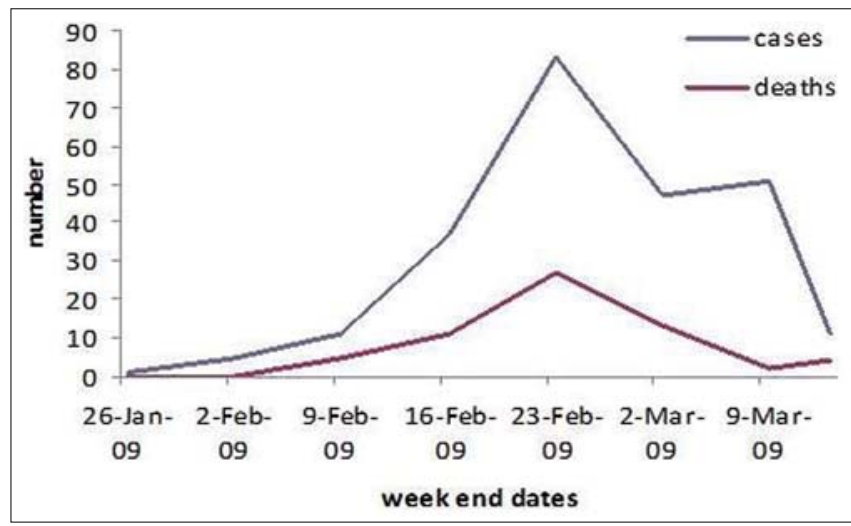

Figure 1: Week-wise distribution of cases and deaths due to hepatitis B, Sabarkantha district, Gujarat, 2009

\begin{tabular}{|c|c|c|}
\hline Variables & Number of cases & Number of deaths \\
\hline \multicolumn{3}{|c|}{ Age group (years) } \\
\hline $0-12$ & 25 & 3 \\
\hline $13-45$ & 409 & 65 \\
\hline$>45$ & 94 & 25 \\
\hline \multicolumn{3}{|l|}{ Gender } \\
\hline Male & 324 & 60 \\
\hline Female & 204 & 33 \\
\hline Total & 528 & 93 \\
\hline
\end{tabular}

\begin{tabular}{|c|c|c|}
\hline & Cases & Controls \\
\hline Exposure to injection in last 6 months & 11 & 3 \\
\hline No exposure & 2 & 23 \\
\hline Total & 13 & 26 \\
\hline
\end{tabular}


5. Periodic training programs on "disinfection practices and safe injection practices" for the private practitioners be arranged so as to keep them updated.

6. IEC activity to focus on health care seekers who demand injections for small ailments like fever, cough, and cold and sometimes demand injections from the doctors.

7. STD forms a special risk group. A project might be undertaken to find the incidence of hepatitis B positivity in HIV positive or other STD-positive cases.

\section{Response of the state/district health authorities}

1. Medical professionals including private practitioners were sensitized for management of hepatitis cases at a continuing medical education (CME) organized with the support of local health professionals.

2. A mass survey was done as a part of active surveillance covering the entire population of the affected talukas of Sabarkantha district covering 1372 villages in the districts.

3. IEC activities were started for sensitizing the community about the disease prevention.

4. Hepatitis B vaccine was given to health care workers and close family contacts of the confirmed cases.

5. A mass vaccination campaign was done with hepatitis $B$ vaccine that covered 77,258 population of Modasa block.

\section{DISCUSSION}

In India, virtually all outbreaks of viral hepatitis are considered to be due to feco-orally transmitted hepatitis non- $\mathrm{A}$ and non-B virus (HEV). ${ }^{[3-5]}$ Outbreaks of viral hepatitis $A$ have also been reported occasionally. ${ }^{[3]}$ Previously, an outbreak of viral hepatitis B had already been reported in the same state Gujarat, India attributed to the use of inadequately sterilized needles and syringes by an unqualified medical practitioner. ${ }^{[6]}$ The present epidemic described here is also attributed to the same practice.

On exploring the history of the patients affected in this outbreak it was found that, out of 528 confirmed cases 210 had given history of receipt of injection in preceding 6 months due to some reason. It is worthwhile to note that out of above 210 recipients of injections, 93 patients had received injection from a single local medical practitioner in Modasa block and out of these 93 cases 51 died due to acute hepatitis $\mathrm{B}$.

Normally acute hepatitis B does not have such a high mortality, but the outbreak reported here was marked by a high case fatality rate of $17.6 \%$, though it was less than that reported in year $1996 .^{[6]}$ In year 1996, the high mortality was attributed to concurrent infection of the cases with hepatitis-D. So, coinfection with HDV was considered as the first possibility for high mortality. However, the virological analysis of the isolated cases neither showed HDV nor other hepatitis viruses such as HAV, HEV, or HCV as the cofactors. However, a higher $(7.7 \%)$ proportion of $\mathrm{HEV}$ coinfections in those presented with fulminant hepatic failure (FHF) patients is noteworthy (though statistically insignificant). ${ }^{[7]}$ Moreover, the hepatitis B virus showed the presence of Pre-Core and Basal Core Mutants, and 4 amino acid substitutions exclusively among FHF cases. None of the self-limiting patients exhibited these dual mutations. Genotype D was predominant, D1 being present in all FHF cases while D2 was most prevalent among cases presenting with acute viral hepatitis. ${ }^{[7]}$

It, therefore, appears that because of a breach in the accepted infection control procedures by the qualified medical practitioners, hepatitis $\mathrm{B}$ infection was more prevalent in the affected area for a reasonable period of time. In addition to this, the gross and continued use of contaminated injection devices (multiple injections with the same needle/syringe from a single vial, reuse of syringes and needles without sterilization, etc.) led to the outbreak. Undoubtedly, other transmission modes would also have added to the epidemic proportion. The identification of association of errors in infection control practices with the epidemic of hepatitis B in a large community alarms all those concerned and highlights need for awareness, education and monitoring of basic infection control practices as well as necessity for formulating strict and well-defined actions against those who violate the norms. The state government did arrange for mass vaccination in affected areas, the impact of which will be visible in due course.

\section{CONCLUSIONS}

Hepatitis B can cause a higher rate of acute infection and outbreak associated with unsafe injection practices, as shown in Sabarkantha district, Gujarat. Sensitization workshop for safe injection practices and waste management need to be conducted among health professionals intermittently to prevent this type of catastrophic outbreaks.

\section{REFERENCES}

1. Aggarwal R, Ranjan P. Preventing and treating hepatitis $B$ infection. Br Med J 2004;329:1080-6.

2. Lai CL, Ratziu V, Yuen MF, Poynard T. Viral hepatitis B. Lancet 2003;362:2089-94.

3. Tandon BN, Gandhi BM, Joshi YK. Etiological spectrum of viral hepatitis and prevalence of markers of hepatitis $A$ and $B$ infection in north India. Bull World Health Organ 1984;62:67-73.

4. Naik SR, Aggarwal R, Salunke PN, Mehrotra NN. A large waterborne viral hepatitis $E$ epidemic in Kanpur, India. Bull World Health Organ 1992;70:597-604.

5. Singh J, Aggarwal NR, Bhattacharjee J, Prakash C, Bora D, Jain DC, et al. An outbreak of viral hepatitis E: Role of community practices. J Commun Dis 1995;27:92-96.

6. Singh J, Bhatia R, Gandhi JC, Kaswekar AP, Khare S, Patel SB, et al. Outbreak of viral hepatitis $B$ in a rural community in India linked to inadequately sterilized needles and syringes. Bull World Health Organ 1998;76:93-8.

7. Arankalle VA, Gandhi S, Lole KS, Chadha MS, Gupte GM, Lokhande MU. An outbreak of hepatitis B with high mortality in India: Association with precore, basal core promoter mutants and improperly sterilized syringes. J Viral Hepat 2011;18:e20-8.

How to cite this article: Dash B, Das RR. Outbreak of hepatitis B in Sabarkantha district of Gujarat: A case-control study. Int J Med Public Health 2013;3:119-21.

Source of Support: Nil, Conflict of Interest: None declared. 